\title{
Mesenchymal Stem Cells Recruit Macrophages to Alleviate Experimental Colitis Through TGF $\beta 1$
}

\author{
Weixin Liu ${ }^{a}$ Shen Zhang ${ }^{a} \quad$ Shouzhi Gub Lixuan Sang ${ }^{c} \quad$ Cong Daia \\ aDepartment of Gastroenterology, First Affiliated Hospital of China Medical University, Shenyang, China; \\ bDepartment of Anatomy Seirei Christopher College, Hamamatsu, Japan; 'Department of Cadre Ward II, \\ First Affiliated Hospital of China Medical University, Shenyang, Liaoning, China
}

\section{Key Words}

Mesenchymal stem cells (MSCs) • Dextran sulfate sodium (DSS) • Ulcerative colitis • TGF $\beta$ receptor signaling $\cdot$ Macrophages

\begin{abstract}
Background/Aims: Transplantation of mesenchymal stem cells (MSCs) has been shown to alleviate dextran sulfate sodium (DSS) -induced colitis through modulation of transforming growth factor $\beta$ (TGF $\beta$ ) receptor signaling. However, the exact molecular mechanisms are not known. Methods: Here, we transplanted primary mouse MSCs or injected TGF $\beta 1$ into mice with DSS-induced colitis. Cells were purified by flow cytometry. Gene expression was analyzed by RT-qPCR. Results: We found that MSCs significantly alleviated the DSS-induced colitis, and the major sources for TGF $\beta 1$ were macrophages that were recruited by MSCs. Specific ablation of macrophages completely abolished the anti-inflammatory effects of MSCs. On the other hand, TGF $\beta 1$ administration, without the presence of MSCs, was sufficient to reduce the severity of DSS-induced colitis. Conclusions: Taken together, our data suggest that MSCS transplantation may recruit macrophages to produce TGF $\beta 1$, which mitigates the pathology of colitis. Thus, MSCs transplantation appears to be a promising therapy for severe enteritis.
\end{abstract}

Copyright $\odot 2015$ S. Karger AG, Basel

\section{Introduction}

Ulcerative colitis and Crohn's disease are two major inflammatory bowel diseases due to pathological immunity. Thus, understanding the molecular mechanisms that regulate the pathogenesis of ulcerative colitis and Crohn's disease may substantially improve

Weixin Liu

KARGER 125
Department of Gastroenterology, First Affiliated Hospital of China Medical University, 155 Nanjing Street, Shenyang 110001 (China)

Tel. +862425138988, E-Mail weixing_liu14@163.com 
their therapy. Among all the experimental animal models developed to investigate these inflammatory bowel diseases, dextran sulfate sodium (DSS) is the most commonly used one, which could be manipulated to resemble either acute or chronic colitis $[1,2]$.

Transforming growth factor $\beta$ (TGF $\beta$ ) receptor signaling has a well-established role in regulating the progression and recovery of inflammation-related colitis [3-8]. However, the precise mechanisms have not been completely clarified. Recently, transplantation of bonemarrow derived cells, or particularly mesenchymal stem cells (MSCs), has been found to reduce the severity of, and improve the recovery from DSS-induced colitis [9-12]. Moreover, modulated TGF $\beta$ receptor signaling by MSCs in the injured colon has been indicated as a cause for the effect of MSCs [12], whereas the exact sources for TGF $\beta$ receptor ligands are not defined, and a direct causal link has not been proved.

Here we transplanted primary mouse MSCs into the isogeneic mice that had developed colitis by DSS administration. We found that MSC transplantation significantly alleviated the DSS-induced colitis. Moreover, significantly higher TGF $\beta 1$ was detected in the colon from the MSC-grafted mice, and was further found to be mainly produced by the recruited macrophages, rather than by MSCs themselves. To investigate whether TGF $\beta 1$ by macrophages is responsible for the effects of MSCs on colitis, we performed a loss-of-function experiment by specific ablation of macrophages with a saporin-conjugated anti-CD11b antibody, which completely abolished the anti-inflammatory effects of MSCs. In a gain-of-function experiment, TGF 1 administration, without the presence of MSCs, was sufficient to reduce the severity of DSS-induced colitis.

\section{Materials and Methods}

\section{Mouse manipulations}

All mouse experiments were approved by the general principles contained in the Guide for the Care and Use of Laboratory Animals published by China Medical University. Male C57BL/6 mice of 10 weeks of age were used for isolation of MSCs, and for all in vivo experiments. Five mice were analyzed in each experimental group. DSS solution (DSS; Sigma-Aldrich, St. Louis, MO, USA) was given to mice via drinking water for 7 days to induce colitis, as has been described before [8, 12]. For in vivo depletion of macrophages,

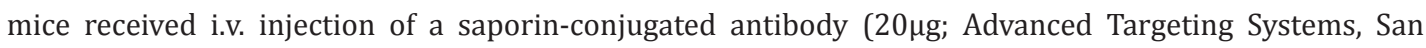
Diego, CA, USA) against the pan-macrophage surface marker CD11b twice per week, as has been described before [13], while the control group received injection of IgG of same frequency. Recombinant TGFß1 was purchased from R\&D System (Los Angeles, CA, USA), and given to mice via intraperitoneal injection twice per week of a dose of $100 \mathrm{ng}$.

\section{Isolation, culturing, labeling and differentiation of MSCS}

The mouse MSCs were isolated and grown in culture as has been described previously [12, 14]. Briefly, plugs of marrow from C57BL/6 mice were dispersed in Dulbecco's Modification of Eagle's Media (DMEM, Life technologies, San Jose, CA, USA) and then centrifuged at 1000g for $4 \mathrm{~min}$. The pellets were re-suspended and plated at $10^{5} \mathrm{cells} / \mathrm{cm}^{2}$ in DMEM containing $10 \% \mathrm{FBS}$. After 10 passages' selection of attached cells, the cells were infected with a lentivirus carrying GFP under a CMV promoter to label with green fluorescence. Transduced cells were purified for GFP (Becton-Dickinson Biosciences, San Jose, CA, USA) by flow cytometry. A positive clone was selected, expanded and then subjected to chondrogenetic, osteogenic, and adipogenic differentiation assays to confirm a MSC phenotype. For chondrogenetic induction, $2.5 \times 10^{5}$

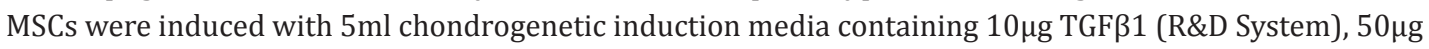
insulin growth factor 1 (R\&D System), and $2 \mathrm{mg} / \mathrm{ml}$ dexamethasone (DMSO, Sigma-Aldrich) followed by centrifugation at $500 \mathrm{~g}$ for $5 \mathrm{~min}$. The cell pellets were maintained in the chondrogenetic induction media for 14 days and subjected to Alcian blue staining. For osteogenic induction, cells were digested and seeded onto a 24-well plate at a density of $10^{4}$ cells/well, and then maintained in osteogenic induction media containing $10 \mathrm{nmol} / \mathrm{l}$ Vitamin D3 (Sigma-Aldrich) and 10mM $\beta$-phosphoglycerol and $0.1 \mu \mathrm{mol} / \mathrm{l}$ DMSO for 14 days and were subjected to Von kossa staining. For adipogenic induction, cells were digested and seeded onto a 24well plate at a density of $10^{4}$ cells/well, and then maintained in the adipogenic induction media containing 
0.5mM 3-isobutyl-1-methylxanthine (IBMX), $200 \mu \mathrm{mol} / \mathrm{l}$ indomethacin, $10 \mu \mathrm{mol} / \mathrm{l}$ insulin and $1 \mu \mathrm{mol} / \mathrm{l}$ DMSO for 14 days and subjected to Oil red 0 staining.

Disease activity index

Disease activity index (DAI) was performed as previously described [8, 12]. Briefly, the scores for body weight loss ( 0 points, $<5 \%$ weight loss; 1 point, $5-10 \%$ weight loss; 2 points, $10-15 \%$ weight loss; 3 points, $15-20 \%$ weight loss; and 4 points, $>20 \%$ weight loss), stool consistency ( 0 points, formed pellets; 2 points, pasty/semiformed stool; and 4 points, liquid stool) and rectal bleeding ( 0 points, no rectal bleeding; and 4 points, visible gross bleeding) were summarized and presented as DAI.

\section{Histology and RT-qPCR}

Colon was excised, freed of adherent tissue and rinsed with ice-cold PBS to remove the fecal material. Segments of colon were then excised, cut, and used for histology, or for mRNA extraction followed by RTqPCR. For histology, colon tissue was fixed in 4\% paraformaldehyde, cut into small pieces, embedded in paraffin, sectioned at $6 \mu \mathrm{M}$, and then stained with Hematoxylin and Eosin (H\&E staining). For RT-qPCR, RNA was extracted from the colon tissue or the sorted cells with Trizol (Invitrogen, St Louis, MO, USA), and then used for cDNA synthesis. RT-qPCR was performed in duplicates with QuantiTect SYBR Green PCR Kit (Qiagen). All primers were purchased from Qiagen. Values of genes were normalized against $\alpha$-tubulin and then compared to the controls.

\section{Flow cytometry}

Digestion of colon cells were performed as described $[8,12]$. Single colon cells were analyzed by flow cytometry after incubation with PEcy5-conjugated F4/80 antibody (Becton-Dickinson Biosciences, San Jose, CA, USA) for isolation of macrophages, and with Lin, Sca-1 and CD49e (Becton-Dickinson Biosciences) for isolation of myofibroblasts. The MSCs were detected by direct GFP. FSC, forward scatter.

\section{Statistical analysis}

Statistical analyses were performed with SPSS 18.0 software (SSPS Inc., Chicago, IL, USA). All data were statistically analyzed using one-way ANOVA with a Bonferoni correction. $\chi 2$ test with Fisher's correction was used for comparison of survival in each experimental group. All values are depicted as mean \pm standard deviation from 5 individuals and are considered significant if $\mathrm{p}<0.05$.

\section{Results}

Isolation, culturing, labeling and differentiation of primary mouse MSCs

Primary mouse MSCs were isolated and expanded in culture, after which the cells were infected with a lentivirus carrying GFP under a CMV promoter to label with green fluorescence (Fig. 1A). Transduced cells were purified by flow cytometry based on GFP (Fig. 1B). A positive clone was selected, expanded and then subjected to differentiation assays including Von kossa staining to evaluate osteogenic induction, Oil red 0 staining to evaluate adipogenic induction and Alcian blue staining to evaluate chondrogenetic induction, which confirmed a MSC phenotype (Fig. 1C-E).

\section{MSCs significantly alleviated DSS-induced colitis}

DSS was given to mice via drinking water for 1 week to induce acute colitis, while mice that received drinking water without DSS were used negative controls (Sham). Afterwards, isogeneic MSCs $\left(10^{6}\right)$ were transplanted into the DSS-treated mice through tail vein injection (DSS+MSCs). Same volume of physiological saline was injected into other DSS-treated mice as positive controls (DSS). All the mice were sacrificed two weeks after MSCs' transplantation (Fig. 2A). We found that the DSS-treated mice significantly increased DAI, compared to Sham, while MSCs transplantation significantly reduced the levels of DAI in DSS-treated mice (Fig. 2B). Moreover, histological analyses showed alleviation of the local inflammation by MSCs transplantation in the DSS-treated mice (Fig. 2C), which were confirmed by the levels of 


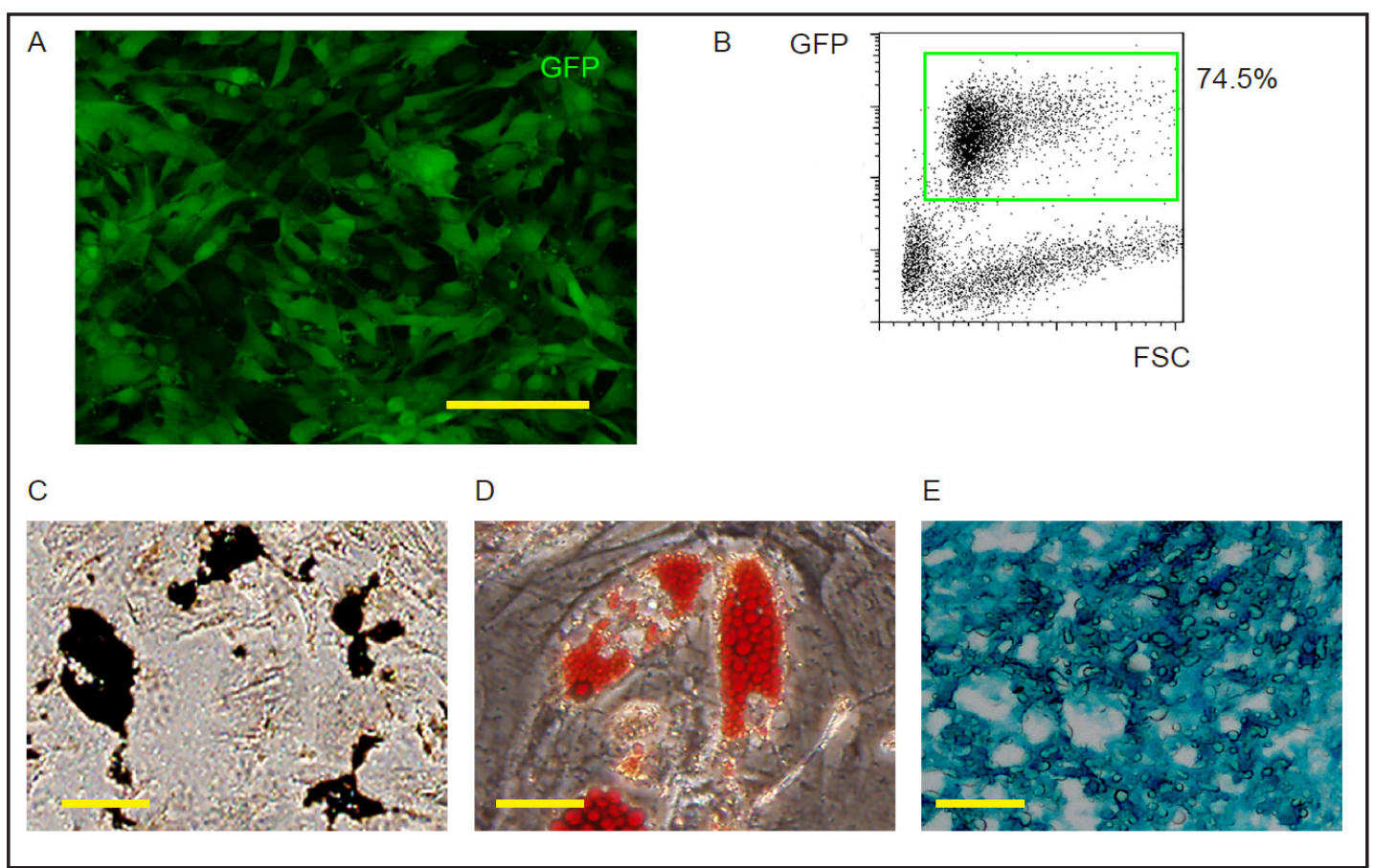

Fig. 1. MSCs culture and differentiation assays. (A) MSCs transduced with lenti-GFP in culture. (B) Purification of transduced GFP-positive MSCs (rectangle) by flow cytometry. (C-E) MSC differentiation assay. (C) Von kossa staining to evaluate osteogenic induction. (D) Oil red $\mathrm{O}$ staining to evaluate adipogenic induction. (E) Alcian blue staining to evaluate chondrogenetic induction. Scale bars are $40 \mu \mathrm{m}$.

inflammation-related factors (Fig. 2D). These data suggest that MSCs significantly alleviated DSS-induced colitis.

MSCs recruited macrophages that produced high levels of TGF $\beta 1$

Since TGF $\beta$ receptor signaling pathway has been shown to promote the recovery of DSS-induced colitis, we thus examined the levels of the major TGF $\beta$ receptor ligand, TGF $\beta 1$, in the colons from Sham, DSS-treated, and DSS-treated, MSCs-grafted mice. We also isolated MSCs (based on GFP) and macrophages (based on F4/80) from the DSS+MSCs mouse lung by flow cytometry (Fig. 3A), and checked TGF $\beta 1$ levels in these purified cells. In order to compare the levels of TGF $\beta 1$ in macrophages to myofibroblasts, we also isolated Lin; Sca$1^{\text {low }}$ and CD49e $\mathrm{e}^{\text {high }}$ myofibroblasts by flow cytometry. We found that MSCs transplantation increased TGF $\beta 1$ levels in the colon from DSS-treated mice by $28.5 \pm 4.0$ fold compared to those in Sham, or by $5.3 \pm 0.7$ fold compared to DSS only treated mice (Fig. 3B). Moreover, the major sources for TGF $\beta 1$ appeared to be the recruited macrophages, rather than by MSCs themselves, since not only macrophages had a more than 2-fold higher TGF $\beta 1$ per cell than myofibrolasts (Fig. 3B), but also a more than 10 -fold higher cell number. These data suggest that MSCs recruited macrophages that produced high levels of TGF $\beta 1$.

Therapeutic effects of MSCs depend on macrophages

To investigate whether TGF $\beta 1$ by macrophages is responsible for the effects of MSCs on colitis, first we performed a loss-of-function experiment by specific ablation of macrophages after MSCs transplantation. We did an i.v. injection of $20 \mu \mathrm{g}$ saporin-conjugated anti-CD11b antibody to the mice twice per week, and compared with the mice received control IgG injection of same frequency (Fig. 4A). We found that ablation of macrophages significantly abolished the anti-inflammatory effects of MSCs, by DAI (Fig. 4B), and by histology (Fig. 4C), suggesting that the therapeutic effects of MSCs depend on macrophages. 


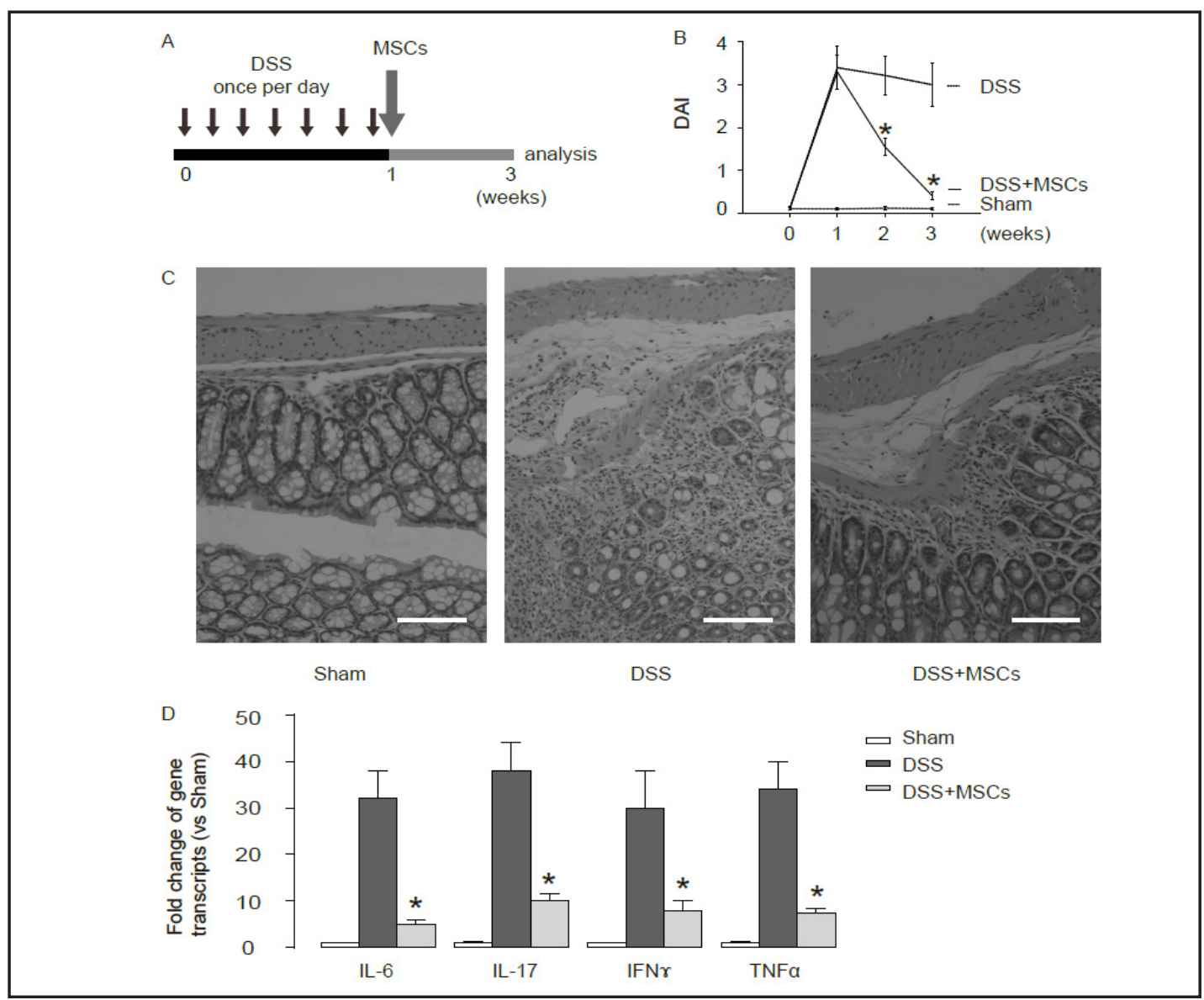

Fig 2. MSCs transplantation alleviated DSS-induced colitis. (A) The schematic of the experiment. DSS was given to mice via drinking water for 1 week to induce acute colitis, while mice that received drinking water without DSS were used negative controls (Sham). Afterwards, isogeneic MSCs $\left(10^{6}\right)$ were transplanted into the DSS-treated mice through tail vein injection (DSS+MSCs). Same volume of physiological saline was injected into other DSS-treated mice as positive controls (DSS). All the mice were sacrificed two weeks after MSCs' transplantation. (B) Disease activity index (DAI) was evaluated in the DSS-treated mice. (C) Histology. (D) RT-qPCR for IL-6, IL-17, IFN $\gamma$ and TNF $\alpha$ in the DSS-treated colon.*: p<0.05; Scale bars are $40 \mu \mathrm{m}$.

\section{TGFB1 is sufficient to reduce the severity of DSS-induced colitis}

Then we examined whether it was TGF $\beta 1$ produced by macrophages that mediated the effects of MSCs on colitis. In a gain-of-function experiment, we gave DSS-treated mice (without MSCs transplantation) an i.p. injection of TGF $\beta 1$ twice per week for 2 weeks (Fig. $4 \mathrm{~A}$ ). We found that TGF $\beta 1$, without the presence of MSCs, was sufficient to reduce the severity of DSS-induced colitis (Fig. 4B-C). Thus, MSCs transplantation may recruit macrophages to produce TGF 31 , which mitigates the pathology of colitis (Fig. 5).

\section{Discussion}

DSS-induced colitis in mice has been shown to be a reproducible animal model for ulcerative colitis in humans. TGF $\beta 1$ receptor signaling has been shown to play an essential role during the pathogenesis and recovery of inflammation-related colitis [3-7], but through unclear mechanism. Also, MSCs transplantation has been reported to promote the recovery from DSS-induced colitis [9-12], whereas the underlying molecular mechanisms remain largely undefined. 
Fig 3. MSCs recruited macrophages that produced high levels of TGF $\beta 1$. Since TGF $\beta$ receptor signaling pathway has been shown to promote the recovery of DSS-induced colitis, we thus examined the levels of the major TGF $\beta$ receptor ligand, TGF $\beta 1$, in the colons from Sham, DSS-treated, and DSS-treated, MSCs-grafted mice. We also isolated MSCs and macrophages (MФ) from the digested DSS+MSCs
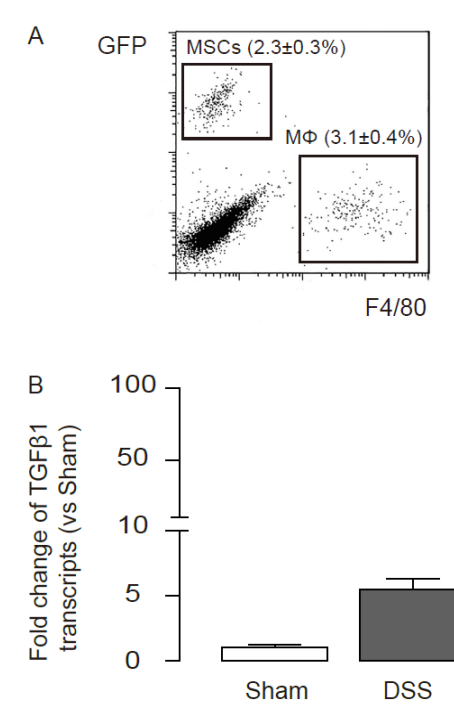

$$
+4 / 80
$$

mouse lung by flow cytometry (A), based on GFP and F4/80, respectively. (B) TGFß1 levels in MSCs, MФ, myofibroblasts and colon tissue from Sham, DSS, and DSC+MSCs -treated mice. *: $\mathrm{p}<0.05$.

Fig 4. Therapeutic effects of MSCs depend on macrophages. (A) Schematic of loss-of-function and gain-of-function experiment. Saporin-conjugated anti-CD11b antibody was provision after MSCs transplantation, twice per week for 2 weeks. Control mice received IgG. DSS-treated mice (without MSCs transplantation) also received injection of TGF $\beta 1$ twice per week for 2 weeks. (B) DAI. (C) Histology. *: p<0.05; Scale bars are $40 \mu \mathrm{m}$.

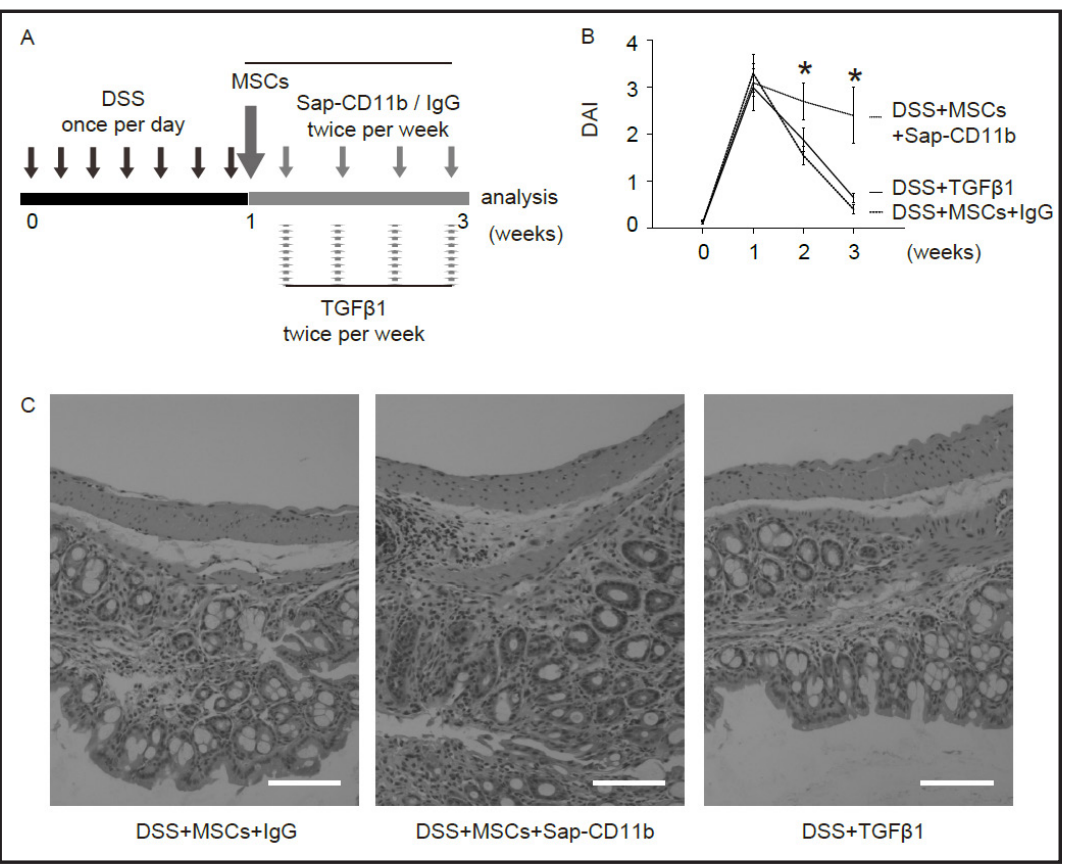

Here, we used a set of loss-of-function and gain-of-function experiments to demonstrate that MSCs transplantation significantly alleviates colitis through augment in TGF $\beta 1$ production by the recruited macrophages. Macrophages are a type of white blood cell that is responsible for phagocytosis in the body. Besides the macrophages with classical phenotype, called "M1" macrophages, another macrophage sub-type, called "M2", is often associated with neovascularization, fibrosis and tissue repair $[12,14-22]$. The conversion between M1 and M2 characteristics for macrophages is called "polarization". Specifically, M2 macrophages are known to secrete a wide range of chemokines, enzymes and growth factors, among which TGF $\beta 1$ is a critical one $[12,19,20]$. Recent studies have shown that MSCs may not only recruit macrophages, but also induce their polarization into a M2 phenotype, in different organs, and in different models $[12,14,19,20]$. Although we did examine the M1/M2 polarization of 
Liu et al.: MSCs Inhibit DSS-Colitis by Macrophage TGF $\beta 1$

Fig 5. Schematic of the model: MSCs transplantation recruits macrophages to produce TGF $\beta 1$, which mitigates the pathology of colitis.

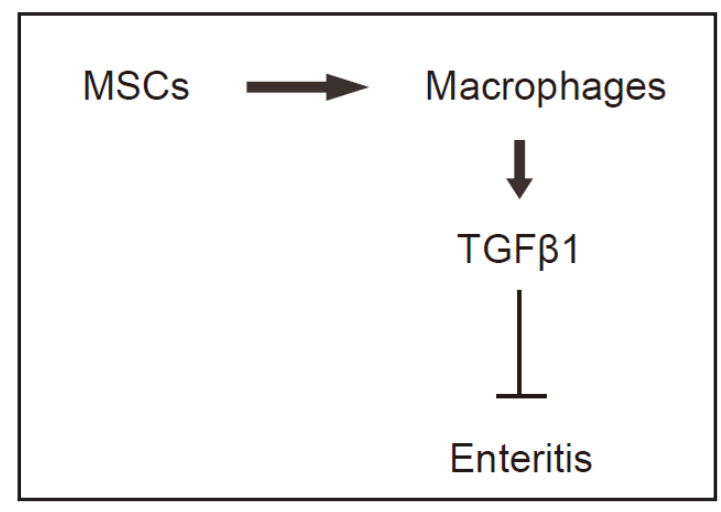

macrophages in the current study, it is highly possible that the functional macrophages here are M2, due to high TGF $\beta 1$ levels in macrophages, and due to the dependence on TGF $\beta 1$ in the MSCs-induced alleviation of the DSS-colitis.

Our findings are consistent with previous reports of the therapeutic effects of MSCs on DSS-induced colitis [12], and further demonstrate the underlying mechanism. Together with previous reports [12], our data highlight a pivotal of macrophage-derived TGF $\beta 1$ in the treatment of ulcerative colitis, suggesting that direct provision of TGF $\beta 1$, or adoptive transfer of M2 macrophages may be promising treatments for ulcerative colitis.

\section{Acknowledgements}

This work was supported by grants from Shenyang Scientific Plan: NO: F13-318-1-40.

\section{Disclosure Statement}

The authors have declared that no competing interests exist.

\section{References}

1 Okayasu I, Hatakeyama S, Yamada M, Ohkusa T, Inagaki Y, Nakaya R: A novel method in the induction of reliable experimental acute and chronic ulcerative colitis in mice. Gastroenterology 1990;98:694-702.

-2 Dieleman LA, Palmen MJ, Akol H, Bloemena E, Pena AS, Meuwissen SG, Van Rees EP: Chronic experimental colitis induced by dextran sulphate sodium (dss) is characterized by th1 and th2 cytokines. Clin Exp Immunol 1998;114:385-391.

3 Hamady ZZ, Scott N, Farrar MD, Wadhwa M, Dilger P, Whitehead TR, Thorpe R, Holland KT, Lodge JP, Carding SR: Treatment of colitis with a commensal gut bacterium engineered to secrete human tgf-beta1 under the control of dietary xylan 1. Inflamm Bowel Dis 2011;17:1925-1935.

4 Duchmann R, Zeitz M: T regulatory cell suppression of colitis: The role of tgf-beta. Gut 2006;55:604-606.

5 Del Zotto B, Mumolo G, Pronio AM, Montesani C, Tersigni R, Boirivant M: Tgf-beta1 production in inflammatory bowel disease: Differing production patterns in crohn's disease and ulcerative colitis. Clin Exp Immunol 2003;134:120-126.

6 Whiting CV, Tarlton JF, Bailey M, Morgan CL, Bland PW: Abnormal mucosal extracellular matrix deposition is associated with increased tgf-beta receptor-expressing mesenchymal cells in a mouse model of colitis. J Histochem Cytochem 2003;51:1177-1189.

7 Neurath MF, Fuss I, Kelsall BL, Presky DH, Waegell W, Strober W: Experimental granulomatous colitis in mice is abrogated by induction of tgf-beta-mediated oral tolerance. J Exp Med 1996;183:2605-2616.

-8 Ye H, Wu Q, Zhu Y, Guo C, Zheng X: Ginsenoside rh2 alleviates dextran sulfate sodium-induced colitis via augmenting tgfbeta signaling. Mol Biol Rep 2014;41:5485-5490. 
-9 Hayashi Y, Tsuji S, Tsujii M, Nishida T, Ishii S, Nakamura T, Eguchi H, Kawano S: The transdifferentiation of bone-marrow-derived cells in colonic mucosal regeneration after dextran-sulfate-sodium-induced colitis in mice. Pharmacology 2007;80:193-199.

10 Tanaka H, Arimura Y, Yabana T, Goto A, Hosokawa M, Nagaishi K, Yamashita K, Yamamoto H, Sasaki Y, Fujimiya M, Imai K, Shinomura Y: Myogenic lineage differentiated mesenchymal stem cells enhance recovery from dextran sulfate sodium-induced colitis in the rat. J Gastroenterol 2011;46:143-152.

11 Tanaka F, Tominaga K, Ochi M, Tanigawa T, Watanabe T, Fujiwara Y, Ohta K, Oshitani N, Higuchi K, Arakawa T: Exogenous administration of mesenchymal stem cells ameliorates dextran sulfate sodium-induced colitis via anti-inflammatory action in damaged tissue in rats. Life Sci 2008;83:771-779.

$\$ 12$ Wang C, Chen J, Sun L, Liu Y: Tgf-beta signaling-dependent alleviation of dextran sulfate sodium-induced colitis by mesenchymal stem cell transplantation. Mol Biol Rep 2014;41:4977-4983.

13 Kanai T, Uraushihara K, Totsuka T, Nemoto Y, Fujii R, Kawamura T, Makita S, Sawada D, Yagita H, Okumura $\mathrm{K}$, Watanabe M: Ameliorating effect of saporin-conjugated anti-cd11b monoclonal antibody in a murine t-cell-mediated chronic colitis. J Gastroenterol Hepatol 2006;21:1136-1142.

14 Cao X, Han ZB, Zhao H, Liu Q: Transplantation of mesenchymal stem cells recruits trophic macrophages to induce pancreatic beta cell regeneration in diabetic mice. Int J Biochem Cell Biol 2014;53:372-379.

15 Flavell RA, Sanjabi S, Wrzesinski SH, Licona-Limon P: The polarization of immune cells in the tumour environment by tgfbeta. Nat Rev Immunol 2010;10:554-567.

16 Gordon S, Taylor PR: Monocyte and macrophage heterogeneity. Nat Rev Immunol 2005;5:953-964.

17 Pollard JW: Trophic macrophages in development and disease. Nat Rev Immunol 2009;9:259-270.

18 Grunewald M, Avraham I, Dor Y, Bachar-Lustig E, Itin A, Jung S, Chimenti S, Landsman L, Abramovitch R, Keshet E: Vegf-induced adult neovascularization: Recruitment, retention, and role of accessory cells. Cell 2006;124:175-189.

19 Xiao X, Gaffar I, Guo P, Wiersch J, Fischbach S, Peirish L, Song Z, El-Gohary Y, Prasadan K, Shiota C, Gittes GK: M2 macrophages promote beta-cell proliferation by up-regulation of smad7. Proc Natl Acad Sci USA 2014;111:E1211-1220.

20 Shen B, Liu X, Fan Y, Qiu J: Macrophages regulate renal fibrosis through modulating tgfbeta superfamily signaling. Inflammation 2014;37:2076-2084.

-21 Song X, Xie S, Lu K, Wang C: Mesenchymal stem cells alleviate experimental asthma by inducing polarization of alveolar macrophages. Inflammation 2014 in press. DOI: 10.1007/s10753-014-9954-9956

22 Xiao Q Zhang X, Wu Y, Yang Y: Inhibition of macrophage polarization prohibits growth of human osteosarcoma. Tumour Biol 2014;35:7611-7616. 